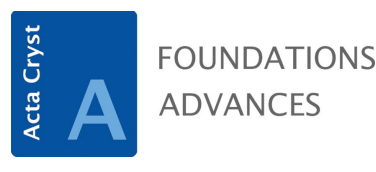

ISSN 2053-2733
Received 16 October 2015

Accepted 15 January 2016

Edited by W. F. Kuhs, Georg-August University Göttingen, Germany

Keywords: Compton scattering; magnetoelectric properties; synchrotron radiation; density functional theory; DFT; Korringa-KohnRostoker (KKR) Green's function method; KKR method.

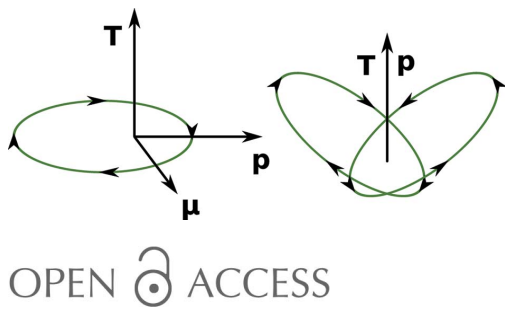

\section{On the possibility of using X-ray Compton scattering to study magnetoelectrical properties of crystals}

\author{
S. P. Collins, ${ }^{a *}$ D. Laundy, ${ }^{a}$ T. Connolley, ${ }^{a}$ G. van der Laan, ${ }^{a}$ F. Fabrizi, ${ }^{a}$ \\ O. Janssen, ${ }^{b}$ M. J. Cooper, ${ }^{c}$ H. Ebert ${ }^{d}$ and S. Mankovsky ${ }^{d}$
}

a Diamond Light Source Ltd, Harwell Science and Innovation Campus, Didcot, OX11 ODE, England, ${ }^{\mathbf{b}}$ Department of Physics, New York University, New York, NY 10003, USA, 'Department of Physics, University of Warwick, CV4 7AL, England, and ' Universität München, Department Chemie, Haus E2.033, Butenandtstrasse 5-13, D-81377 München, Germany. ${ }^{*}$ Correspondence e-mail: steve.collins@diamond.ac.uk

This paper discusses the possibility of using Compton scattering - an inelastic $\mathrm{X}$-ray scattering process that yields a projection of the electron momentum density - to probe magnetoelectrical properties. It is shown that an antisymmetric component of the momentum density is a unique fingerprint of such time- and parity-odd physics. It is argued that polar ferromagnets are ideal candidates to demonstrate this phenomenon and the first experimental results are shown, on a single-domain crystal of $\mathrm{GaFeO}_{3}$. The measured antisymmetric Compton profile is very small $\left(\simeq 10^{-5}\right.$ of the symmetric part) and of the same order of magnitude as the statistical errors. Relativistic first-principles simulations of the antisymmetric Compton profile are presented and it is shown that, while the effect is indeed predicted by theory, and scales with the size of the valence spin-orbit interaction, its magnitude is significantly overestimated. The paper outlines some important constraints on the properties of the antisymmetric Compton profile arising from the underlying crystallographic symmetry of the sample.

\section{Background}

Compton scattering provides a projection of the electron momentum distribution in a target material (Cooper, 1985). While the exact relativistic form of the differential scattering cross section is complex, the momentum density derived from every measurement, and calculated by every theory, to date, has been symmetric. We argue that this is because all materials investigated so far have been symmetric with respect to time reversal or spatial inversion. Materials whose orbitals possess neither symmetry are said to be magnetoelectric as they play a major role in magnetoelectric phenomena. Of particular interest are toroidal moments, corresponding to time- and parity-odd vectors, that not only play a vital role in magnetoelectric phenomena (Spaldin et al., 2008) but have been suggested to be implicated in high- $T_{\mathrm{c}}$ superconductivity (Scagnoli et al., 2011).

It is therefore of considerable interest to identify novel experimental probes of such moments. We show that the antisymmetric Compton profile is a unique signature of magnetoelectric properties and should therefore provide a very sensitive probe of the underlying orbitals, that can be compared in detail to electronic structure calculations to elucidate the underlying physics. In this article, we outline the principles behind this phenomenon, examine the possibility of observing such an effect in the polar ferromagnetic crystal $\mathrm{GaFeO}_{3}$, describe an experiment to measure the antisym- 
metric Compton profile, and compare the results with relativistic first-principles calculations.

\section{Compton scattering and the electron momentum density}

$\mathrm{X}$-ray Compton scattering is an inelastic scattering process whereby the energy loss is an almost linear function of a projection of the electron momentum density:

$$
\frac{\mathrm{d}^{2} \sigma}{\mathrm{d} \Omega \mathrm{d} E} \propto J\left(p_{z}\right)
$$

where

$$
J\left(p_{z}\right)=\iint_{p_{x}} \int_{p_{y}} \rho(\mathbf{p}) \mathrm{d} p_{x} \mathrm{~d} p_{y}
$$

is called the Compton profile (Cooper, 1985). Here, $\mathbf{z}$ lies (almost) parallel to the momentum transfer $\mathbf{q}^{\prime}-\mathbf{q}$ and $p_{z}=\mathbf{p} \cdot \hat{\mathbf{z}}$ is the $z$ projection of electron momentum. The momentum density $\rho(\mathbf{p})$ and Compton profile $J\left(p_{z}\right)$ are historically considered to be symmetric with respect to reversal of the momentum variable $\mathbf{p} \rightarrow-\mathbf{p}$ (or $p_{z} \rightarrow-p_{z}$ ). We suggest that this need not be the case.

Let us first discuss the conditions under which the momentum density is symmetric. Since momentum is a function of both space and time (classically, $p \propto \mathrm{d} \mathbf{r} / \mathrm{d} t$ ) we find that either inverting $\mathbf{r}$ (parity inversion) or $t$ (time reversal) inverts $\mathbf{p}(\mathbf{p} \rightarrow-\mathbf{p})$. Consequently, any parity-even (centrosymmetric) or time-even (non-magnetic) material must satisfy $\rho(\mathbf{p})=\rho(-\mathbf{p})$. Compton profiles of this dominant class of materials are always symmetric.

However, no such constraint applies to materials that lack both time and inversion symmetry. Moreover, such systems form an interesting and important class of materials that often exhibit magnetoelectric phenomena such as linear magnetoelectric coupling, destined to play a key role in future technologies (Spaldin \& Fiebig, 2005). We are therefore alerted to the possibility of using Compton scattering as a probe of timeand parity-odd magnetoelectric phenomena.

It is worth noting that while the breakdown of the impulse approximation (IA) can lead to an asymmetry in the measured Compton profile (Huotari et al., 2001), such effects need not be considered for the current analysis. This is partly because our measurements are not of the asymmetry in the energy spectrum directly, but rather of the intensity difference that is caused by an asymmetry in the electron momentum distribution. Moreover, the orbitals that are expected to contribute to the effect discussed here are relatively low-energy valence states, whereas the breakdown of the IA is expected to affect mainly tightly bound core electrons.

It is convenient to write the total momentum density as the sum of a symmetric component (with respect to $\mathbf{p} \rightarrow-\mathbf{p}$ ) and an antisymmetric part:

$$
\rho(\mathbf{p})=\rho_{\mathrm{S}}(\mathbf{p})+\rho_{\mathrm{A}}(\mathbf{p})
$$

giving

$$
J\left(p_{z}\right)=J_{\mathrm{S}}\left(p_{z}\right)+J_{\mathrm{A}}\left(p_{z}\right)
$$

where

$$
J\left(p_{z}\right)_{S(A)}=\int_{p_{x}} \int_{p_{y}} \rho(\mathbf{p})_{S(A)} \mathrm{d} p_{x} \mathrm{~d} p_{y} .
$$

The quantities $\rho_{\mathrm{A}}(\mathbf{p})$ and $J_{\mathrm{A}}\left(p_{z}\right)$ represent time- and parityodd properties. Since Compton scattering is an incoherent process, these objects are averages over all the constituent orbitals and therefore governed by the crystal (magnetic) point-group symmetry.

\section{Zero-sum rule}

If there is no net flow of electrons in the sample the integral of the flow along positive and negative $z$ directions must cancel, i.e.

$$
\int_{-\infty}^{\infty} p_{z} J\left(p_{z}\right) \mathrm{d} p_{z}=0
$$

While this is satisfied trivially for the symmetric Compton profile, it imposes a useful constraint on each half of the antisymmetric profile:

$$
\int_{0}^{\infty} p_{z} J_{\mathrm{A}}\left(p_{z}\right) \mathrm{d} p_{z}=0
$$

thus providing a 'zero-sum rule' that can be used to verify experimental results and model calculations. Most trivially, the zero-sum rule dictates that, for each half of the asymmetric profile, the existence of a positive contribution implies the existence of a negative one, and vice versa.

\section{Feasibility argument: classical orbitals}

Finding no obvious symmetry arguments for momentum densities and Compton profiles to be symmetric is, of course, far from demonstrating that they are not. However, one can

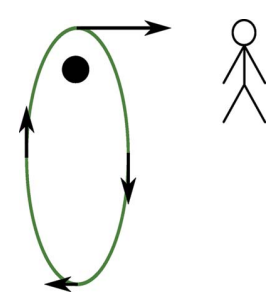

Figure 1

A gedankenexperiment to demonstrate the possibility of a non-symmetric momentum density in a classical orbital. An observer sits in the plane of a highly eccentric planetary orbit, perpendicular to its major axis, and with the planet moving towards the observer at perihelion. The observer measures the amount of time each projection of the planet's momentum is observed for (perhaps via a Doppler-shift measurement) during a complete orbit, observing a large positive momentum projection for a small amount of time, and a small negative momentum for a long time as the planet orbits furthest from the star. The largest positive momentum has no negative counterpart and so the momentum density is clearly asymmetric. It is clear that this function is reversed under reversal of time (i.e. the planet orbits in reverse), and also under spatial inversion, realized (in this two-dimensional system) by a rotation of $\pi$ of the orbital within the orbital plane. 
employ a simple thought experiment to see that such an asymmetry is present in classical orbitals. Consider a highly elliptical planetary orbital, observed from within the orbital plane, as shown in Fig. 1. The orbiting body would be seen to have a very large positive momentum projection (towards the observer) for a short period of time, when the orbiting 'planet' is closest to the 'star' that it orbits. Conversely, it would exhibit a small negative momentum projection for a long period of time when it is far from the star and moving slowly. The large positive momentum has no negative counterpart and so the momentum projection distribution (analogous to the Compton profile) must be asymmetric. Note also that such an orbital is asymmetric with respect to time reversal and spatial inversion: reversing time would reverse the direction of the orbit, and spatial inversion would reverse its eccentricity.

\section{Momentum density and rotational properties}

The electron momentum density is a real-valued function of momentum, p, or (equivalently) of its magnitude, $p$, and direction, $\hat{\mathbf{p}}$. We can expand this density in terms of a complete set of angular functions and prefactors that depend on $p$. For example,

$$
\rho(\mathbf{p})=\sum_{K=0}^{\infty} \sum_{Q=-K}^{K} T_{Q}^{K}(p) Y_{Q}^{K}(\hat{\mathbf{p}})
$$

where $Y_{Q}^{K}$ are real spherical harmonics (also referred to as multipoles or spherical tensors) of rank $K$ and projection $Q$ (Lovesey et al., 2005), and $T_{Q}^{K}(p)$ are the corresponding tensor components and are functions of $p$. The merit of such an expansion lies in the fact that each non-vanishing multipole $Y_{Q}^{K}$ must be consistent with the symmetry of the physical system. For example, an isotropic system allows only a single term in the expansion and we find

$$
\rho(\mathbf{p})=T_{0}^{0}(p)=\frac{I(p)}{4 \pi p^{2}}
$$

where $I(p)$ is the radial momentum distribution (Cooper, 1985).

For the present study we are primarily interested in the antisymmetric momentum density:

$$
\rho_{\mathrm{A}}(\mathbf{p})=\sum_{K=0}^{\infty} \sum_{Q=-K}^{K} A_{Q}^{K}(p) Y_{Q}^{K}(\hat{\mathbf{p}})
$$

where $A_{Q}^{K}(p)$ are the corresponding tensor components. Since reversal of the momentum vector, $\mathbf{p} \rightarrow-\mathbf{p}$, is equivalent to carrying out the rotations $Y_{Q}^{K}(\theta, \varphi) \rightarrow Y_{Q}^{K}(\pi-\theta, \pi+\varphi)$, and all $K=$ odd (even) real spherical harmonics are odd (even) under this transformation, we conclude that the antisymmetric momentum density contains only $K=$ odd terms, ruling out contributions from magnetic monopoles or quadrupoles. (Conversely, the symmetric density contains only even multipoles, including the scalar $K=0$ term). We can therefore write

$$
\rho_{\mathrm{A}}(\mathbf{p})=\sum_{K=1,3,5 \ldots}^{\infty} \sum_{Q=-K}^{K} A_{Q}^{K}(p) Y_{Q}^{K}(\hat{\mathbf{p}}) .
$$

Importantly, the lowest-order allowed component has $K=1$ and is therefore associated with a parity-odd, time-odd vector, i.e. a toroidal moment (Lovesey et al., 2005). A non-vanishing antisymmetric Compton profile therefore requires a material whose point-group symmetry permits the existence of oddrank time- and parity-odd multipoles. One might expect systems that allow the lowest $(K=1)$ rank multipole to be most favourable. If we assume that the antisymmetric momentum density is dominated by this term then we have

$$
\rho_{\mathrm{A}}(\mathbf{p}) \simeq \sum_{Q=-1}^{1} A_{Q}^{1}(p) Y_{Q}^{1}(\hat{\mathbf{p}}) .
$$

Furthermore, if the direction of the toroidal moment is fixed by symmetry (i.e. the same for all momentum magnitudes) then the radial and angular parts can be factorized:

$$
\rho_{\mathrm{A}}(\mathbf{p}) \simeq A^{1}(p) \sum_{Q=-1}^{1} a_{Q}^{1} Y_{Q}^{1}(\hat{\mathbf{p}})
$$

$\left(a_{Q}^{1}\right.$ are now constants), which can be written in Cartesian form as

$$
\rho_{\mathrm{A}}(\mathbf{p}) \simeq A^{1}(p) \hat{\mathbf{T}} \cdot \hat{\mathbf{p}}
$$

where $\hat{\mathbf{T}}$ is the toroidal moment direction.

The Compton profile of such a momentum density can be written:

$$
\begin{aligned}
& J_{\mathrm{A}}\left(p_{z}\right) \simeq \int_{p_{x}} \int_{p_{y}} A^{1}(p) \hat{\mathbf{T}} \cdot \hat{\mathbf{p}} \mathrm{d} p_{x} \mathrm{~d} p_{y} \\
& =\iint_{p_{x}} \frac{A^{1}(p)}{p}\left\{p_{x} \hat{\mathbf{T}} \cdot \hat{\mathbf{x}}+p_{y} \hat{\mathbf{T}} \cdot \hat{\mathbf{y}}+p_{z} \hat{\mathbf{T}} \cdot \hat{\mathbf{z}}\right\} \mathrm{d} p_{x} \mathrm{~d} p_{y} \\
& =\int_{p_{x}} \int_{p_{y}} \frac{A^{1}(p)}{p}\left\{p_{x} \hat{\mathbf{T}} \cdot \hat{\mathbf{x}}\right\} \mathrm{d} p_{x} \mathrm{~d} p_{y}+\iint_{p_{x}} \int_{p_{y}} \frac{A^{1}(p)}{p}\left\{p_{y} \hat{\mathbf{T}} \cdot \hat{\mathbf{y}}\right\} \mathrm{d} p_{x} \mathrm{~d} p_{y} \\
& \quad+\int_{p_{x}} \int_{p_{y}} \frac{A^{1}(p)}{p}\left\{p_{z} \hat{\mathbf{T}} \cdot \hat{\mathbf{z}}\right\} \mathrm{d} p_{x} \mathrm{~d} p_{y} .
\end{aligned}
$$

The first two terms vanish as they involve integrals over functions that are odd with respect to the integration variable. This leaves

$$
\begin{aligned}
J_{\mathrm{A}}\left(p_{z}\right) & \simeq \int_{p_{x}} \int_{p_{y}} \frac{A^{1}(p)}{p}\left\{p_{z} \hat{\mathbf{T}} \cdot \hat{\mathbf{z}}\right\} \mathrm{d} p_{x} \mathrm{~d} p_{y} \\
& =p_{z} \hat{\mathbf{T}} \cdot \hat{\mathbf{z}} \int_{p_{x}} \int_{p_{y}} \frac{A^{1}(p)}{p} \mathrm{~d} p_{x} \mathrm{~d} p_{y} .
\end{aligned}
$$

While the integral is not straightforward to compute, there are two noteworthy aspects of this result. First, the Compton profile changes sign with $p_{z} \rightarrow-p_{z}$, as expected. More interestingly, the Compton profile scales with the $z$ projection of the toroidal moment unit vector. The latter gives a complete description of the angular dependence of the antisymmetric 
Table 1

Allowed vectors and their directions, for various magnetic symmetry groups $(+/-$ indicates the absence/presence of the time-reversal operator) and vector symmetry with respect to time $(T)$ and parity $(P)$.

The Cartesian axes $x, y, z$ are parallel to the crystal axes $a, b, c$. The third of the four listed groups, $m^{\prime} m 2^{\prime}$ (conventionally written $m^{\prime} 2^{\prime} m$ ), corresponds to the symmetry of $\mathrm{GaFeO}_{3}$.

\begin{tabular}{llllll}
\hline Symmetry & & & & & Vector \\
name & Symmetry operation & $T$ & $P$ & Vector type & direction \\
\hline$m^{\prime} m^{\prime}$ & $\left\{1+, 2_{y}-, m_{x}+, m_{z}-\right\}$ & + & + & Axial (time-even) & 0 \\
& $\left\{1+, 2_{y}-, m_{x}+, m_{z}-\right\}$ & + & - & Polar & $y$ \\
& $\left\{1+, 2_{y}-, m_{x}+, m_{z}-\right\}$ & - & + & Magnetic & $x$ \\
& $\left\{1+, 2_{y}-, m_{x}+, m_{z}-\right\}$ & - & - & Toroidal & $z$ \\
& & & & & \\
$m^{\prime} m^{\prime} 2$ & $\left\{1+, 2_{y}+, m_{x}-, m_{z}-\right\}$ & + & + & Axial (time-even) & 0 \\
& $\left\{1+, 2_{y}+, m_{x}-, m_{z}-\right\}$ & + & - & Polar & $y$ \\
& $\left\{1+, 2_{y}+, m_{x}-, m_{z}-\right\}$ & - & + & Magnetic & $y$ \\
& $\left\{1+, 2_{y}+, m_{x}-, m_{z}-\right\}$ & - & - & Toroidal & 0 \\
$m^{\prime} m 2^{\prime}$ & $\left\{1+, 2_{y}-, m_{x}-, m_{z}+\right\}$ & + & + & Axial (time-even) & 0 \\
& $\left\{1+, 2_{y}-, m_{x}-, m_{z}+\right\}$ & + & - & Polar & $y$ \\
& $\left\{1+, 2_{y}-, m_{x}-, m_{z}+\right\}$ & - & + & Magnetic & $z$ \\
& $\left\{1+, 2_{y}-, m_{x}-, m_{z}+\right\}$ & - & - & Toroidal & $x$ \\
& & & & & \\
$m m 2$ & $\left\{1+, 2_{y}+, m_{x}+, m_{z}+\right\}$ & + & + & Axial (time-even) & 0 \\
& $\left\{1+, 2_{y}+, m_{x}+, m_{z}+\right\}$ & + & - & Polar & $y$ \\
& $\left\{1+, 2_{y}+, m_{x}+, m_{z}+\right\}$ & - & + & Magnetic & 0 \\
& $\left\{1+, 2_{y}+, m_{x}+, m_{z}+\right\}$ & - & - & Toroidal & $y$ \\
& & & &
\end{tabular}

Compton profile in the case where multipoles of $K \geq 3$ are negligible: the signal is maximum when the toroidal moment is along $z$, of equal and opposite magnitude when antiparallel, and zero when perpendicular. Armed with this approximate form, and the exact 'zero-sum rule' in equation (7), a clear picture begins to emerge about what the antisymmetric Compton profile should look like.

\section{Experimental verification - suitable materials}

It is likely that the antisymmetric part of the Compton profile is small as it depends on a subtle aspect of the anisotropy of the orbital polarization. An ideal experiment to test these ideas is therefore one where the antisymmetric part can be reversed simply and rapidly, inducing the smallest possible systematic error and allowing a sensitive 'difference' measurement to be performed. Reversal of the toroidal vector in many magnetoelectric materials requires simultaneous application of electric and magnetic fields, typically applied during 'field cooling'. An interesting class of materials where the toroidal moments are more easily manipulated are polar ferromagnets. One such material, that has been studied with $\mathrm{X}$-rays for its directional dichroism (Kubota et al., 2004) and magnetoelectric multipoles (Staub et al., 2010), is $\mathrm{GaFeO}_{3}$. Large polar single crystals are available and the ferromagnetic moment can be reversed with a modest applied field. $\mathrm{GaFeO}_{3}$ orders magnetically at the relatively high temperature of $T_{\mathrm{C}} \simeq$ $210 \mathrm{~K}$. We therefore selected $\mathrm{GaFeO}_{3}$ as a potentially suitable test material and consider next the implications of crystal symmetry on the observable physical phenomena.

\section{7. $\mathrm{GaFeO}_{3}$ : symmetry and allowed tensor components}

$\mathrm{GaFeO}_{3}$ (space-group No. 33, $P c 2_{1} n$ ) is a polar ferromagnet. It possesses both a magnetic (axial, time-odd) and polar (timeeven) vector moment. We have discussed the need for timeand parity-odd multipoles in the context of antisymmetric Compton profiles, and the desirability to possess a toroidal (polar, time-odd) moment. We now apply the magnetic crystal symmetry to all four permutations of time/parity odd/even vectors in order to find out (i) if they can exist and (ii) in which direction(s) they may point. The crystal point-group symmetry in the high-temperature paramagnetic phase is $m m 2$. We can denote the symmetry group as $\left\{1,2_{y}, m_{x}, m_{z}\right\}$ (the identity, twofold rotation about $y$, mirrors normal to $x$ and $z$ ). There are several possible magnetic groups that are consistent with this point group, which are formed by taking each spatial symmetry operator and either applying time reversal or not. Four such groups can be generated, with each placing specific constraints on the directions of the possible vectors, or rendering them absent.

For $\mathrm{GaFeO}_{3}$, it is known that the magnetic easy axis lies along $x$ (crystal $a$ axis). However, the anisotropy is not strong and it is informative to consider the properties of all possible magnetic symmetry groups. These are shown in Table 1 . The procedure for analysing the properties of various tensors, permitted by crystal symmetry, is described in Collins \& Bombardi (2010). Briefly, the process involves generating a random vector, odd or even under $T$ (time) and $P$ (parity), generating a transformed vector for each of the four symmetry operations in the magnetic group, and adding the four resulting vectors. We find that the resultant, due to the high symmetry of the system, is always either zero or lies parallel to one of the three Cartesian/crystal axes.

Several interesting points emerge from this symmetrization procedure. First, we see that in all cases the time-even polar vector lies along $y$. This makes sense as only the magnetic symmetry is changed between the four groups. We see that there is no time-even axial vector. The four magnetic groups support a magnetic vector along $x, y$ and $z$ for the first three, with the fourth group not supporting a net magnetic moment.

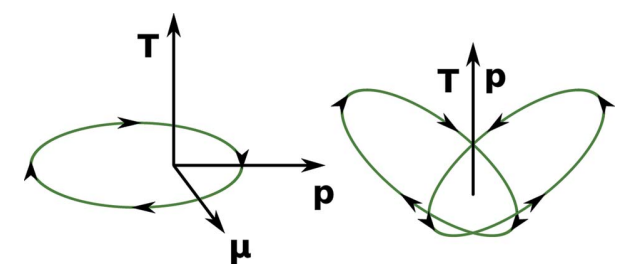

Figure 2

Left: a classical representation of the dipole (vector) moments permitted by a classical orbital. The magnetic moment $\mu$ points out of the page and is perpendicular to the orbital plane. The polar (electric dipole) moment, $\mathbf{p}$, lies parallel to the major orbital axis. The toroidal moment, T, lies perpendicular to the polar and magnetic moments, in the direction of their cross-product, and is thus odd under reversal of either, but even under reversal of both. Right: the slightly counter-intuitive case of zero magnetic moment and parallel polar and toroidal moments can be visualized classically as the superposition of two rotated and reflected orbitals, forming a 'butterfly' pattern. 


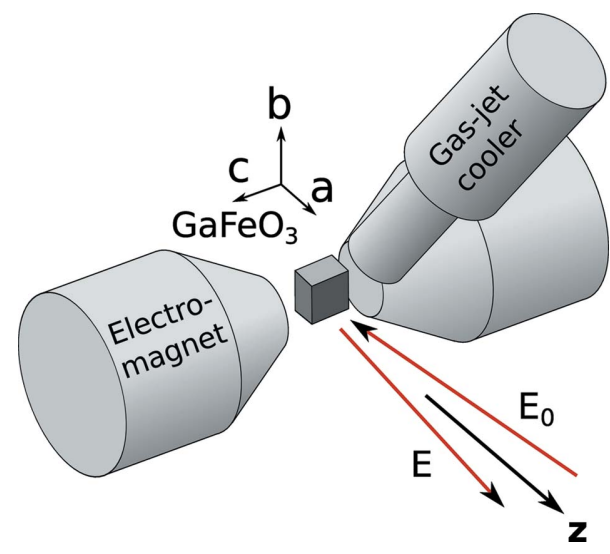

Figure 3

A schematic depiction of the experimental setup designed for measuring the antisymmetric Compton profile in a crystal of the polar ferromagnet $\mathrm{GaFeO}_{3}$. A reversible magnetic field was applied along the crystal $c$ axis, with the polar $b$ axis vertical. The toroidal $a$ axis was aligned close to the direction of photon momentum transfer, which defines the projection direction for the momentum density. The sample was held below its magnetic ordering temperature by a nitrogen gas-jet cooler, and scattered photons detected by a multi-element germanium solid-state detector array (not shown), close to back-scattering.

Of particular interest are the toroidal vectors. For the first and third groups (the homomorphic $m m^{\prime} 2^{\prime}$ and $m^{\prime} m 2^{\prime}$ groups), the toroidal vector is perpendicular to the magnetic and polar vector, consistent with the sketch in Fig. 1. The second group $\left(m^{\prime} m^{\prime} 2\right)$ does not allow a toroidal vector. Interestingly, a toroidal vector is allowed by the fourth group ( $\mathrm{mm} 2)$, despite the absence of a magnetic moment, and it is parallel to the polar vector. This slightly counter-intuitive scenario can be visualized as the sum of two such classical orbitals, resembling a butterfly (Fig. 2).

All four symmetry groups support $K=3$ and higher (odd) rank multipoles. For the present case, where the magnetic field is applied along the magnetic easy axis (the first magnetic group in the table), the presence of a toroidal moment suggests that $\mathrm{GaFeO}_{3}$ is a suitable material for observing an antisymmetric Compton profile, and that the $c$-axis toroidal moment should be directed along the momentum transfer $(z$ axis), with the polar $b$ axis and magnetic $a$ axis both perpendicular, as shown in Fig. 3.

\section{Experiment on $\mathrm{GaFeO}_{3}$}

Experiments were carried out on beamline I12 (Diamond Light Source), using a linearly polarized monochromatic incident X-ray beam of energy $125 \mathrm{keV}$ and bandwidth of $0.6 \mathrm{keV}$, selected by controlled bending of a double Laue monochromator (Drakopoulos et al., 2015). Compton scattering was detected close to back-scattering $\left(2 \theta \sim 169^{\circ}\right)$ by a 23-element germanium solid-state detector. The orientation of crystal (a single polar domain - see Appendix $A$ ), X-ray beams and magnetic field were as shown in Fig. 3 and the sample was maintained at a temperature of $100 \mathrm{~K}$ with a nitrogen gas-jet cooler. As the aim of the experiment was to observe a small (antisymmetric) difference in the Compton profiles measured

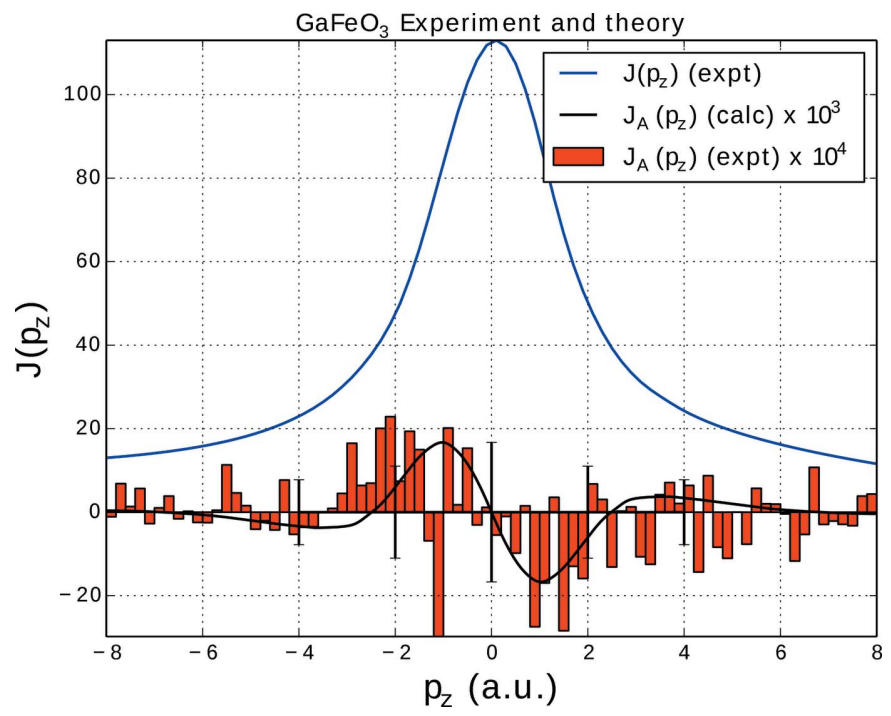

Figure 4

Experimental Compton scattering results from $\mathrm{GaFeO}_{3}$. The total electron momentum distribution (Compton profile) is shown in blue, normalized such that the integral is the total number of electrons in the unit cell (eight $\mathrm{GaFeO}_{3}$ formula units). The red bars show the antisymmetric Compton profile derived from the difference in Compton profiles measured with opposite magnetic field directions. Error bars are shown in black. Also shown on the plot is the calculated antisymmetric profile, convoluted with a Gaussian of width 0.8 a.u., to mimic the experimental momentum resolution. Note that, although the calculated and measured line shapes look similar, the experimental differences are of the same order as the statistical errors (error bars), and that the measurements are scaled by an order of magnitude compared to the calculations.

with two opposite magnetic field directions, the $(0.3 \mathrm{~T})$ field was flipped rapidly and repeatedly ( $1 \mathrm{~s}$ counting time for each direction) while data were accumulated for around $48 \mathrm{~h}$.

The experimental Compton scattering results are shown in Fig. 4. The total Compton scattering (electron momentum density) is shown in blue, with the magnetic 'difference' signal indicated by red bars. The difference data have been multiplied by $10^{4}$, indicating that the difference, and any competing systematic and random errors, are extremely small. While the difference profile gives a hint of the anticipated antisymmetric shape, the effect is of the same order as the statistical errors (black bars). As such, the experimental results do not show conclusive evidence of the predicted asymmetry but give a clear indication of the maximum magnitude of such an effect.

\section{First-principles calculations on $\mathrm{GaFeO}_{3}$}

To confirm the occurrence of an antisymmetric Compton profile in polar ferromagnets, density functional theory (DFT)-based theoretical investigations have been performed. The Compton profile was calculated from first principles using the Korringa-Kohn-Rostoker (KKR) Green's function method. This implies the electronic Green's function $G\left(\mathbf{r}, \mathbf{r}^{\prime}, E\right)$ is represented by means of the multiple scattering formalism by 


$$
\begin{aligned}
G\left(\mathbf{r}, \mathbf{r}^{\prime}, E\right)= & \sum_{\Lambda \Lambda^{\prime}} Z_{\Lambda}^{q}(\mathbf{r}, E) \tau_{\Lambda \Lambda^{\prime}}^{n q n^{\prime} q^{\prime}}(E) Z_{\Lambda^{\prime}}^{q^{\prime} \times}\left(\mathbf{r}^{\prime}, E\right) \\
& -\sum_{\Lambda}\left[Z_{\Lambda}^{q}(\mathbf{r}, E) J_{\Lambda}^{q \times}\left(\mathbf{r}^{\prime}, E\right) \Theta\left(r^{\prime}-r\right)\right. \\
& \left.+J_{\Lambda}^{q}(\mathbf{r}, E) Z_{\Lambda}^{q \times}\left(\mathbf{r}^{\prime}, E\right) \Theta\left(r-r^{\prime}\right)\right] \delta_{n n^{\prime}} \delta_{q q^{\prime}} .
\end{aligned}
$$

Here $\tau_{\Lambda \Lambda^{\prime}}^{n q n^{\prime} q^{\prime}}(E)$ is the scattering path operator with the combined index $\Lambda=(\kappa, \mu)$ representing the spin-orbit and magnetic quantum numbers $\kappa$ and $\mu$, respectively (Rose, 1961), and $Z_{\Lambda}^{q}$ and $J_{\Lambda}^{q}$ are the four-component regular and irregular solutions, respectively, to the single-site Dirac equation for the atomic site $q$ (Ebert et al., 2011). The superscript $\times$ indicates the left-hand-side solution of the Dirac equation. The electron momentum density $\rho(\mathbf{p})=$ $\rho^{\uparrow}(\mathbf{p})+\rho^{\downarrow}(\mathbf{p})$ is decomposed into its spin-projected components $\rho^{\uparrow(\downarrow)}(\mathbf{p})$, which are given by the Green's function represented in momentum space,

$$
\rho^{m_{\mathrm{s}}}(\mathbf{p})=-\frac{1}{\pi} \int_{0}^{E_{F}} \Im G_{m_{\mathrm{s}}}(\mathbf{p}, \mathbf{p}, E) \mathrm{d} E,
$$

where $m_{\mathrm{s}}$ represents the spin character. $G_{m_{\mathrm{s}}}\left(\mathbf{p}, \mathbf{p}^{\prime}, E\right)$ is expressed in terms of the real-space Green's function $G\left(\mathbf{r}, \mathbf{r}^{\prime}, E\right)$ as follows:

$$
\begin{aligned}
& G_{m_{\mathrm{s}}}\left(\mathbf{p}, \mathbf{p}^{\prime}, E\right) \\
& =\frac{1}{N \Omega} \int \mathrm{d}^{3} r \int \mathrm{d}^{3} r^{\prime} \Phi_{\mathbf{p} m_{\mathrm{s}}}^{\times}(\mathbf{r}) \Im G^{+}\left(\mathbf{r}, \mathbf{r}^{\prime}, E\right) \Phi_{\mathbf{p}^{\prime} m_{\mathrm{s}}}\left(\mathbf{r}^{\prime}\right) .
\end{aligned}
$$

Here $\Omega$ is the volume of the unit cell and $\Phi_{\mathbf{p} m_{\mathrm{s}}}$ are the eigenfunctions of the momentum operator, which can be written as $\Phi_{\mathbf{p} m_{\mathrm{s}}}=U_{\mathbf{p} m_{\mathrm{s}}} \exp (i \mathbf{p r})$, where $U_{\mathbf{p} m_{\mathrm{s}}}$ is a fourcomponent spinor satisfying the equation (Rose, 1961)

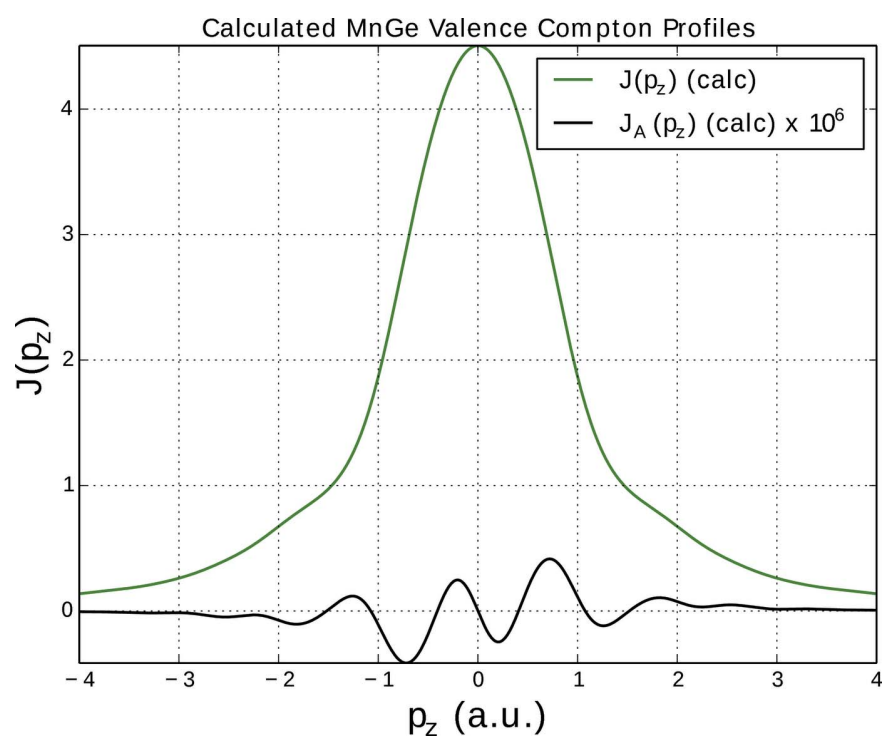

Figure 5

Calculated total valence electron (green) and antisymmetric (black) Compton profiles of the chiral magnet (parity- and time-odd) $\mathrm{MnGe}$, broadened by 0.2 a.u. Calculations are done for the ferromagnetically ordered system with a magnetic field direction parallel to $[1,-1,0]$ and momentum transfer vector $\mathbf{q} \|[1,1,-2]$.

$$
\left(c \boldsymbol{\alpha p}+\beta m c^{2}\right) U_{\mathbf{p} m_{\mathrm{s}}}=E_{p} U_{\mathbf{p} m_{\mathrm{s}}} .
$$

Using a Rayleigh-like expression, one obtains the angular momentum expansion for the eigenfunctions (Benea et al., 2006),

$$
\begin{aligned}
\Phi_{\mathbf{p} m_{\mathrm{s}}}= & 4 \pi\left(\frac{E_{p}+c^{2}}{2 E_{p}+c^{2}}\right)^{1 / 2} \sum_{\Lambda} i^{l} C_{\Lambda}^{m_{\mathrm{s}}} Y_{l}^{\mu-m_{\mathrm{s}} *}(\hat{p}) \\
& \times\left[\begin{array}{c}
j_{l}(p r) \chi_{\Lambda}(\mathbf{r}) \\
\frac{i c s_{k}}{E_{p}+c^{2}} j_{l}(p r) \chi_{-\Lambda}(\hat{r})
\end{array}\right],
\end{aligned}
$$

where $C_{\Lambda}^{m_{\mathrm{s}}}$ are Clebsch-Gordan coefficients, $Y_{l}^{m_{l}}$ are complex spherical harmonics, $\chi_{\Lambda}(\hat{r})$ are spin-angular functions and $j_{l}(p r)$ are spherical Bessel functions.

The electronic structure calculations have been performed using the fully relativistic multiple scattering KKR Green's function method (Ebert et al., 2011, 2012) (adopting the atomic sphere approximation, ASA). Exchange and correlation were treated within the framework of local spin density approximation (LSDA) using the parametrization of Vosko, Wilk and Nusair (Vosko et al., 1980). Chemical disorder due to intermixing between the $\mathrm{Fe}$ and Ga sublattices in the system is treated by means of the coherent potential approximation (CPA) alloy theory (Soven, 1967; Ebert et al., 2011). For the angular momentum expansion of the Green's function [see equation (17)] a cutoff of $\ell_{\max }=3$ was applied.

As a first step in the investigations of the occurrence of the antisymmetric Compton profile, the calculations have been performed for the non-centrosymmetric compounds $\mathrm{MnGe}$ and $\mathrm{FeGe}$, with B20 structure (space group $P 2_{1} 3$ ). FeGe is ferromagnetically ordered at ambient pressure with a Curie temperature $T_{\mathrm{C}}=278.2 \mathrm{~K}$ (Wilhelm et al., 2012), while MnGe can be synthesized under a high pressure and exhibits antiferromagnetic (AFM) order below $T_{\mathrm{N}}=197 \mathrm{~K}$ with a saturated magnetic moment of $\sim 1.9 \mu_{\mathrm{B}} / \mathrm{Mn}$ at $5 \mathrm{~K}$ (Kanazawa et al., 2011). Despite that, the calculations for both compounds have been performed for an FM (ferromagnetic) alignment of the magnetic moments to fulfil the precondition for the observation of an antisymmetric Compton profile. The calculated Mn magnetic moment in MnGe, of $2.1 \mu_{\mathrm{B}}$, fits rather well the experimental results. In line with the experimental setup, the orientation of the magnetization was taken to be perpendicular to the sample threefold axis as well as to the momentum transfer vector q. For both systems the antisymmetric part of the calculated Compton profile is very weak (but still significant), as is demonstrated in Fig. 5 showing the results for MnGe.

These results are in line with the results of the measurements on MnSi with B20 structure, performed with the same geometry and demonstrating the magnitude of the antisymmetric Compton profile that is beyond the current accuracy of the experiment.

In the case of the $\mathrm{GaFeO}_{3}$ system the calculations were performed taking the occupation numbers 0.18 and 0.35 for $\mathrm{Fe}$ atoms on $\mathrm{Ga} 1$ and $\mathrm{Ga} 2$ sites $[\mathrm{Fe}(\mathrm{Ga} 1)$ and $\mathrm{Fe}(\mathrm{Ga} 2)]$ and 0.77 and 0.7 for the $\mathrm{Fe} 1$ and $\mathrm{Fe} 2$ (Fe sites), respectively. The structure and composition information was taken from the 
available experimental data. The $\mathrm{Fe}$ magnetic moments obtained in the calculations are $M_{\mathrm{Fe}(\mathrm{Ga} 1)}=2.2 \mu_{\mathrm{B}}, M_{\mathrm{Fe}(\mathrm{Ga} 2)}=$ $2.9 \mu_{\mathrm{B}}, M_{\mathrm{Fe} 1}=3.0 \mu_{\mathrm{B}}$ and $M_{\mathrm{Fe} 2}=2.8 \mu_{\mathrm{B}}$, with the $\mathrm{Fe}(\mathrm{Ga} 1)$ and Fe1 magnetic moments antiferromagnetically oriented to $\mathrm{Fe}(\mathrm{Ga} 2)$ and $\mathrm{Fe} 2$, respectively.

The calculations of the antisymmetric Compton profile have been performed for the geometry as used in the experiment. This implies that the photon momentum transfer is along the toroidal axis $a$, while the magnetic field lies parallel to the crystal $c$ axis. Assuming that the total magnetic moment follows the direction of the magnetic field, the contribution of the valence electrons to the Compton profile has been calculated for two opposite orientations $( \pm)$ of the magnetization. Accordingly, the antisymmetric Compton profile $J_{\mathrm{A}}\left(p_{z}\right)$ is defined as the difference $J_{\mathrm{A}}\left(p_{z}\right)=J^{+}\left(p_{z}\right)-J^{-}\left(p_{z}\right)$. Restricting the calculations to the valence electrons implies that contributions to $J_{\mathrm{A}}\left(p_{z}\right)$ by core electrons are negligible. This simplification is very well justified. The total Compton profile due to the valence states is shown in Fig. 6 together with the antisymmetric Compton profile, calculated using a momentum broadening of 0.2 and 0.8 a.u. The latter value corresponds to the experimental momentum broadening. As can be seen, the amplitude of the antisymmetric profile is about three orders of magnitude smaller than for the total Compton profile. In the experiment this difference is even more pronounced, as can be seen in Fig. 4. To account for the rather low experimental momentum resolution of about 0.8 a.u., a corresponding momentum broadening has been applied to the calculated Compton profiles, shown in Fig. 4 (black line). This results in particular in a substantial decrease of the amplitude bringing the theoretical results closer to the experiment. Another source for the apparent overestimation of the antisymmetric

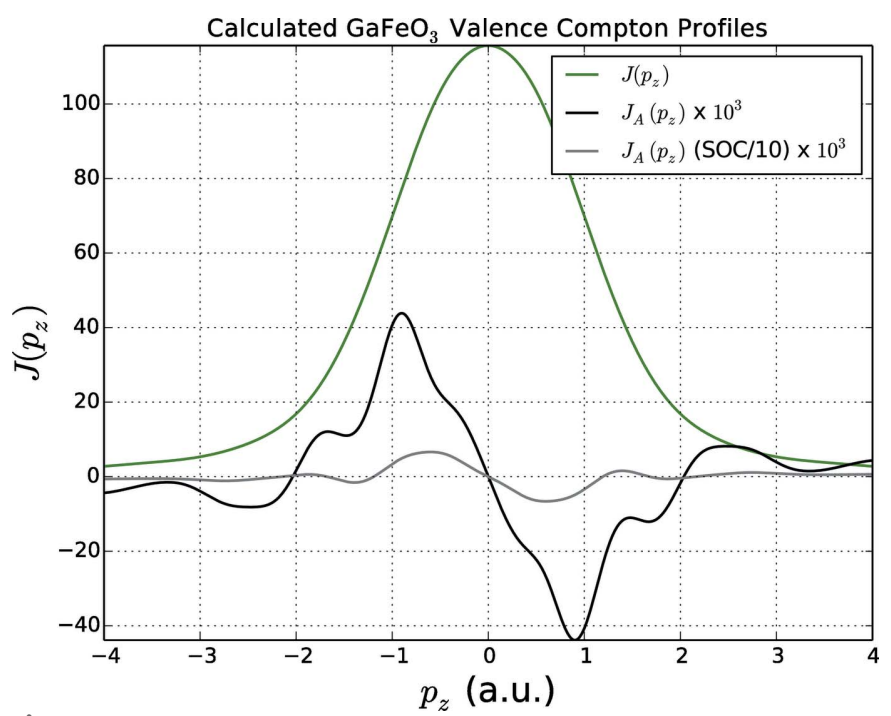

Figure 6

Calculated $\mathrm{GaFeO}_{3}$ total valance electron (green) and antisymmetric (black) Compton profiles, broadened by 0.2 a.u. (a quarter of the momentum resolution of the present work), indicating the potential benefit of performing measurements with improved resolution. Also shown is the same antisymmetric profile, but calculated with the spinorbit coupling (SOC) reduced by a factor of ten. The antisymmetric profile is reduced by very nearly the same factor, showing that SOC plays an essential role in the underlying physics. part of the Compton profile in the calculations is the finite temperature of the measurements $(T=100 \mathrm{~K})$. Taking into account the rather small critical temperature $T_{\mathrm{c}} \simeq 200 \mathrm{~K}$, one can expect a rather pronounced temperature-induced magnetic disorder in the system which should lead to a smearing of the electronic states and as a result to a decrease of the magnitude of the antisymmetric Compton profiles.

As the antisymmetric Compton profile is a consequence of the anisotropy of the orbital polarization and accordingly is first of all a relativistic effect, it should depend on the strength of spin-orbit coupling (SOC) in the system. To demonstrate this, the calculations have been performed with the SOC scaled. In Fig. 6 the antisymmetric profile obtained using a scaling factor $\zeta_{\text {sOC }}=0.1$ is plotted together with that obtained without any SOC scaling. One can clearly see a decrease of the amplitude of the profile by nearly one order of magnitude due to the SOC scaling. A further decrease of the scaling factor leads to a collapse of the antisymmetric part of the Compton profile.

Note also that spin-orbit interaction has a rather pronounced effect on the shape of the magnetic Compton profile $(\mathrm{MCP})$ - the spin-projected momentum density. Fig. 7 gives the MCP for $\mathrm{GaFeO}_{3}$ calculated without (green line) and with (red line) SOC. As one can see, neglect of the SOC results in an increase of the amplitude at $p_{z}=0$ a.u., as well as a more pronounced oscillatory momentum dependence.

\section{Conclusions and future prospects}

We propose a new class of Compton scattering experiment with the potential to provide the antisymmetric part of the electron momentum density in materials. We show that the antisymmetric Compton profile is a unique fingerprint of timeand parity-odd properties of the underlying orbitals, and thus a sensitive probe of magnetoelectric phenomena. Initial

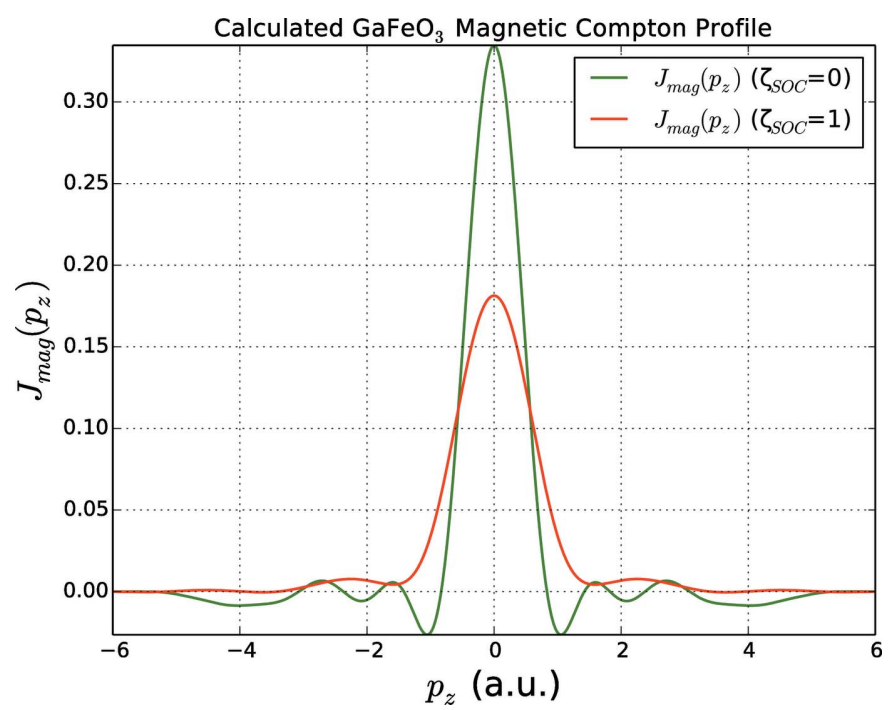

Figure 7

Magnetic Compton profile for $\mathrm{GaFeO}_{3}$ : red line with full SOC and green line with SOC suppressed. A momentum broadening of 0.2 a.u. has been applied. Both profiles have been normalized to the total magnetic moment of $0.27 \mu_{\mathrm{B}}$ per formula unit. 


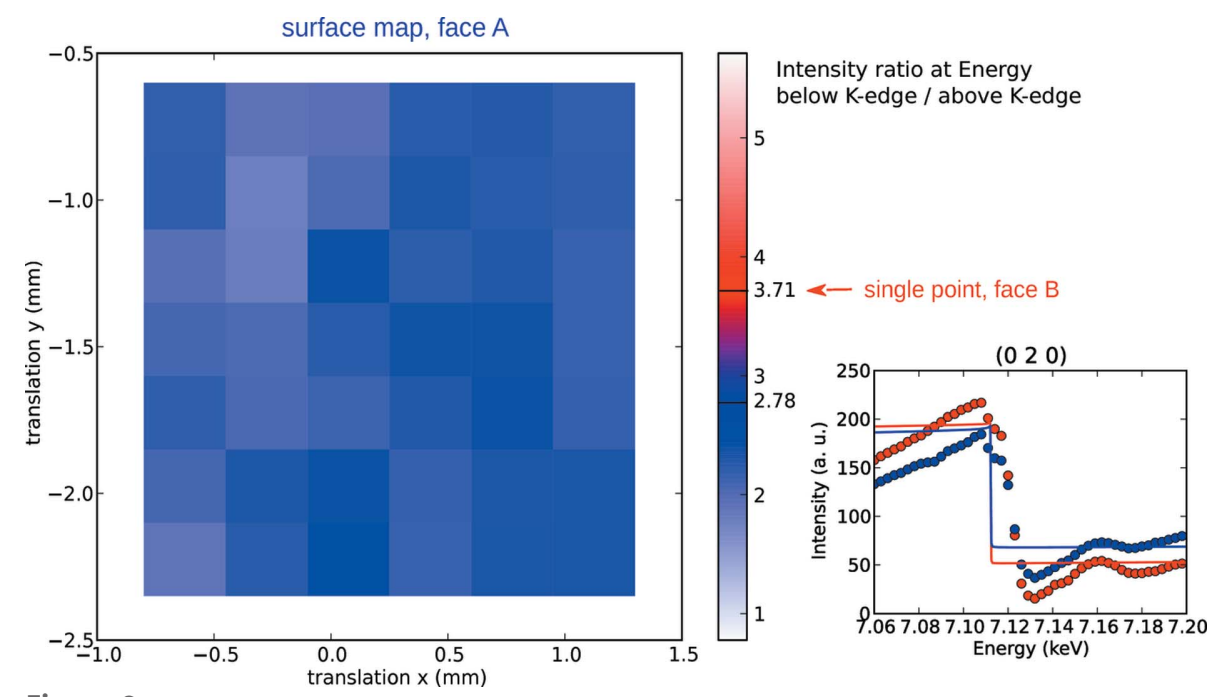

Figure 8

Left: a map of the intensity ratio between photon energies 7.10 and $7.16 \mathrm{keV}$ (below and above the Fe $K$ edge), on the ( 020 ) reflection, for one sample face. The red arrow indicates the intensity ratio for the opposite face, in the central position of the map. The values 2.78 and 3.71, highlighted in the colour bar, represent the expected ratios of a monodomain state, for the two faces, respectively. Right: the complete energy profiles of the reflection, measured at the centre of the map on the two opposite faces (red and blue). The dots represent experimental data (integrated rocking curves); the lines are simulated from a simple model of resonant diffraction from isolated $\mathrm{Fe}^{3+}$ ions.

To conclude, we have shown that antisymmetric Compton scattering should exist in an interesting and topical class of material. First attempts to measure this have shown that it is extremely small and close to the limit of statistical uncertainty, but differs sufficiently from predictions of state-of-theart first-principles calculations, to provide a very sensitive test of the microscopic origins of magnetoelectric phenomena. We expect that future improvements in experimental technology will make such measurements more straightforward. The present study focuses on a polar ferromagnet. While such a system affords simple control over magnetoelectric polarization, via magnetic field flipping, we note that this is by no means necessary. Antisymmetric components of the electron momentum density should be observable in materials that are odd under time and parity reversal sepa-

experiments on the polar ferromagnet $\mathrm{GaFeO}_{3}$ demonstrate that our experimental technique is extremely sensitive, leading to very small systematic errors. Our results show that the magnitude of the antisymmetric momentum density, after broadening with the experimental momentum resolution of 0.79 a.u., is not larger than around $10^{-5}$ of the peak in the symmetric part. While the optimistic eye might be tempted to pick out an antisymmetric difference signal (Fig. 4) above the statistical noise, we cannot claim that the present results are conclusive in this respect.

The main scientific motivation for these experiments is to provide a sensitive and stringent test of first-principles electronic structure calculations. To this end, we have performed calculations of the antisymmetric Compton profile using the KKR Green's function method. The results of these calculations suggest that the antisymmetric profile should be far larger than was observed in the measurements, thus proving that the antisymmetric Compton profile is indeed a highly challenging and stringent test of competing theories. Moreover, even without comparison to experimental data, deficiencies in the theory are evident from the fact that the calculated profiles clearly (visually) violate our zero-sum rule.

Deficiencies in the experimental determination of this effect are even more dramatic than those of the theory. The X-ray detection efficiency, determined by the total detector solid angle, is $\sim 6 \times 10^{-3}$. Moreover, the difference signal is inevitably washed out by convolution with the momentum resolution, determined by the incident photon beam bandwidth, and detector energy and angular resolution. Technological developments, especially high-resolution high-energy photon detector arrays, are likely to improve the quality of experimental data by a very significant factor, rendering such studies straightforward in the future. rately, but even under the combination of the two, such as an antiferromagnetic/antiferroelectric crystal.

Finally, it is perhaps worth noting that Compton scattering has the potential to probe other exotic polarization-dependent properties. For example, one could envisage a study of surface states in a topological insulator, using the spin sensitivity of magnetic Compton scattering (Cooper, 1985) to probe the correlation between the momentum vector of the wavefunction and its spin direction. Such experiments would be significantly more challenging than the present one, due to the required surface sensitivity and the reduced cross section for magnetic Compton scattering, but might be feasible in the future.

\section{APPENDIX $A$}

Verification of the single polar domain state in the $\mathrm{GaFeO}_{3}$ crystal

Since the antisymmetric Compton profile would be expected to vanish if the X-ray beam sampled an equal population of opposite polar domains, the experiment hinged on being confident that the entire crystal, of dimensions $\sim 3 \times 3 \times$ $3 \mathrm{~mm}$, consisted of a single polar domain.

As the crystal was far too large to establish its polar properties by conventional X-ray diffraction techniques, we employed a novel approach, described by Fabrizi et al. (2015), whereby spatial diffraction maps are made of opposite sample faces at two photon energies, just above and below the Fe $K$ edge.

First, the detailed energy dependence of the (ll 200$)$ reflection was collected between 7.06 and $7.2 \mathrm{keV}$, in the central position of the surface, for the two opposite faces. It is 
expected, given the geometry of the system, that reversing the face of the sample equates to reversing the sign of the polar vector, if the crystal is in a monodomain state. This is confirmed by the difference in the two energy profiles (Fig. 8). The contrast between polar states is provided by the resonant contribution of the X-ray diffraction, which is enhanced in the proximity of an atomic absorption edge.

To verify that the monodomain state extends to the whole sample, a spatial map was collected on one of the faces, by measuring the intensity ratio between two opportune photon energies $(7.10$ and $7.16 \mathrm{keV})$. This provides us with a fingerprint of the domain composition, irrespective of the overall scattering power of the specific portion of the crystal illuminated by the X-rays.

These measurements confirmed that each face exhibited a single polar domain, and that the polar vector reversed between opposite faces. It therefore seems extremely likely that the entire crystal is formed from a single polar domain.

\section{Acknowledgements}

GvdL and HE would like to acknowledge the EU-COST action MP1306 (EUSpec). The authors acknowledge Diamond Light Source for time on Beamline I12 under Proposal EE8629.

\section{References}

Benea, D., Mankovskyy, S. \& Ebert, H. (2006). Phys. Rev. B, 73, 94411.
Collins, S. P. \& Bombardi, A. (2010). Magnetism and Synchrotron Radiation: New Trends, Springer Proceedings in Physics, pp. 223261. Berlin, Heidelberg: Springer.

Cooper, M. J. (1985). Rep. Prog. Phys. 48, 415-481.

Drakopoulos, M. et al. (2015). J. Synchrotron Rad. 22, 828-838.

Ebert, H., Ködderitzsch, D. \& Minár, J. (2011). Rep. Prog. Phys. 74, 096501.

Ebert, H. et al. (2012). The Munich SPR-KKR Package. Version 6.3. http://olymp.cup.uni-muenchen.de/ak/ebert/SPRKKR.

Fabrizi, F., Thomas, P. A., Nisbet, G. \& Collins, S. P. (2015). Acta Cryst. A71, 361-367.

Huotari, S., Hämäläinen, K., Manninen, S., Issolah, A. \& Marangolo, M. (2001). J. Phys. Chem. Solids, 62, 2205-2213.

Kanazawa, N., Onose, Y., Arima, T., Okuyama, D., Ohoyama, K., Wakimoto, S., Kakurai, K., Ishiwata, S. \& Tokura, Y. (2011). Phys. Rev. Lett. 106, 156603.

Kubota, M., Arima, T., Kaneko, Y., He, J. P., Yu, X. Z. \& Tokura, Y. (2004). Phys. Rev. Lett. 92, 137401.

Lovesey, S. W., Balcar, E., Knight, K. S. \& Rodríguez, J. F. (2005). Phys. Rep. 411, 233.

Rose, M. E. (1961). Relativistic Electron Theory. New York: Wiley. http://openlibrary.org/works/OL3517103W/Relativistic_electron_ theory.

Scagnoli, V., Staub, U., Bodenthin, Y., de Souza, R. A., GarcíaFernández, M., Garganourakis, M., Boothroyd, A. T., Prabhakaran, D. \& Lovesey, S. W. (2011). Science, 332, 696-698.

Soven, P. (1967). Phys. Rev. 156, 809-813.

Spaldin, N. A. \& Fiebig, M. (2005). Science, 309, 391-392.

Spaldin, N. A., Fiebig, M. \& Mostovoy, M. (2008). J. Phys. Condens. Matter, 20, 434203.

Staub, U., Bodenthin, Y., Piamonteze, C., Collins, S. P., Koohpayeh, S., Fort, D. \& Lovesey, S. W. (2010). Phys. Rev. B, 82, 104411.

Vosko, S. H., Wilk, L. \& Nusair, M. (1980). Can. J. Chem. 58, 12001211.

Wilhelm, H., Baenitz, M., Schmidt, M., Naylor, C., Lortz, R., Rössler, U. K., Leonov, A. A. \& Bogdanov, A. N. (2012). J. Phys. Condens. Matter, 24, 294204. 